\title{
Correction to: Towards Automatic Measurement of Type B Aortic Dissection \\ Parameters: Methods, Applications and Perspective
}

Jianning Li, Long Cao, W. Cheng, M. Bowen, and Wei Guo

\author{
Correction to: \\ Chapter "Towards Automatic Measurement of Type B \\ Aortic Dissection Parameters: Methods, Applications \\ and Perspective" in: D. Stoyanov et al. (Eds.): \\ Intravascular Imaging and Computer Assisted Stenting \\ and Large-Scale Annotation of Biomedical Data \\ and Expert Label Synthesis, LNCS 11043, \\ https://doi.org/10.1007/978-3-030-01364-6_8
}

The original version of the chapter starting on p. 64 was revised. The author names and their affiliations have been changed. Instead of

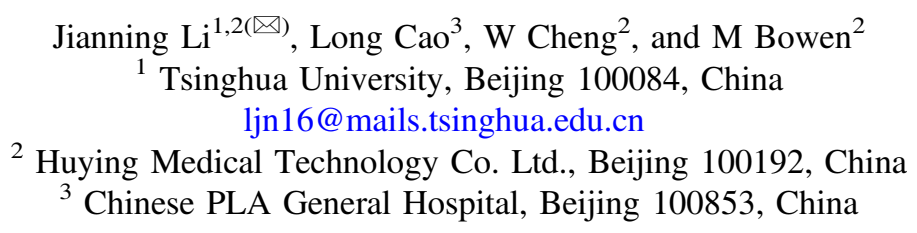

they should be read as follows:

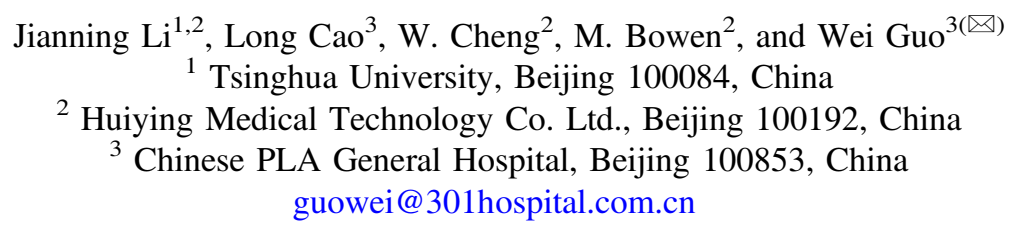

The original chapter was corrected.

The updated version of this chapter can be found at https://doi.org/10.1007/978-3-030-01364-6_8 